MATEC Web of Conferences 11, 02003 (2014)

DOI: $10.1051 /$ matecconf $/ 20141102003$

(C) Owned by the authors, published by EDP Sciences, 2014

\title{
Flexural behavior of reinforced concrete beams: Comparative analysis between high-performance concrete and ordinary concrete
}

\section{Comportement en flexion de poutres en béton armé: Analyse comparée entre béton à hautes performances et béton ordinaire}

\author{
Mostefa Hamrat ${ }^{1}$, Bensaid Boulekbache ${ }^{1}$, Mohamed Chemrouk ${ }^{2}$ et Sofiane Amziane ${ }^{3}$ \\ ${ }^{1}$ Département de Génie Civil, Université Hassiba Benbouali, Chlef, Algérie \\ ${ }^{2}$ Université des Sciences et Technologie Houari Boumediene, Alger, Algérie \\ ${ }^{3}$ Polytech'Clermont, Université Blaise Pascal, Clermont Ferrand, France
}

\begin{abstract}
This paper presents an experimental study on the flexural strength of reinforced concrete beams made with high performance concrete (HPC) and ordinary concrete (OC). We are carried an experimental campaign aimed comes in three points: 1- the study of the law of behavior of the two materials (OC and HPC), 2- the influence of the compressive strength of concrete and the rate of longitudinal reinforcement on the loaddeflection behavior and ductility index, 3-comparative analysis (ACI318, Eurocode 2 and BS8110) against the crack opening. Test results showed that the capacity of the beams in HPC is higher ( $6 \%$ to $20 \%)$ than the beams in OC. The use of HPC is more efficient than the OC to delay the first cracking. The average value of the ductility index for the beams in HPC is 1.30 times those beams in OC. The formula for calculating the crack opening derived of the Eurocode 2 gives the best prediction the crack width of beams (for both types of concrete).
\end{abstract}

\begin{abstract}
Résumé. Ce papier présente une étude expérimentale sur la résistance en flexion de poutres en béton armé faites en béton à hautes performances (BHP) et en béton ordinaire (BO). Nous avons mené une campagne expérimentale dont l'objectif se décline en trois points: 1- l'étude de la loi de comportement des deux matériaux (BO et $\mathrm{BHP}$ ), 2- l'influence de la résistance en compression de béton et le taux d'armature longitudinale sur le comportement charge-flèche et l'indice de ductilité, 3- l'analyse comparative des règlements $(\mathrm{ACI} 318$, Eurocode 2 et $\mathrm{BS} 8110)$ vis à vis de l'ouverture des fissures. Les résultats d'essais ont montré que la capacité portante des poutres en BHP est élevée (6\% à $20 \%$ ) que celle des poutres en BO. L'emploi du BHP est plus efficace que celui du BO pour retarder l'apparition de la fissuration. La valeur moyenne de l'indice de ductilité pour les poutres en BHP est 1,30 fois celles des poutres en BO. La formule permettant le calcul de l'ouverture des fissures issue du règlement de l'Eurocode 2 prédit le mieux l'ouverture des fissures des poutres (pour les deux types de bétons).
\end{abstract}

\section{Introduction}

Le béton à hautes performances (BHP) possède des propriétés physico-mécaniques grandement améliorées par comparaison au béton ordinaire (BO). En fait, le BHP a la réputation d'être plus fragile que le béton ordinaire (BO) [1]. Il s'agit d'une caractéristique matérielle déduite sur des éprouvettes de béton non armé.

Toute étude de matériaux de construction doit non seulement prendre en considération l'amélioration de leurs caractéristiques physico-mécaniques, mais aussi décrire correctement son comportement structurel à savoir la rigidité flexionnelle, la capacité portante et la ductilité à la rupture. Dans ce contexte, nous avons mené une campagne expérimentale dont l'objectif de déterminer dans quelle mesure le comportement structurel d'un BHP diffère-t-il de celui d'un BO, du fait que le matériau BHP présente non seulement une faible résistance en traction par rapport à sa résistance en compression, mais également une fragilité plus grande une fois le pic de charge atteint [2]. 


\section{Programme expérimental}

Deux types de bétons ont été confectionnés, un béton ordinaire $(\mathrm{BO})$ et un béton à hautes performances (BHP). Les formulations sont récapitulées dans le Tableau 1.

Le programme expérimental est basé sur deux séries de poutres selon la résistance du béton. Chaque série comporte trois poutres rectangulaires de section $10 \times 16$ $\mathrm{cm}^{2}$ et de $1.50 \mathrm{~m}$ de portée.

-Série B40, fabriquée avec un béton de $40 \mathrm{MPa}$

-Série B80, fabriquée avec un béton de $85 \mathrm{MPa}$.

Les taux de renforcement (As/bd) des poutres dans la partie tendue correspondant aux diamètres $10 \mathrm{~mm}, 12 \mathrm{~mm}$ et $14 \mathrm{~mm}$ sont respectivement $1.22 \%, 1.77 \%$ et $2.42 \%$.

Les poutres sont placées sur un bâti (machine Zwick/Roell) approprié pour être soumises à la flexion «quatre points» (figure 1).

Une caméra numérique a été utilisée pour suivre l'évolution des fissures lors des essais. L'analyse d'image se fait avec un logiciel Gom-Aramis qui nous permet d'obtenir le champ de déformation sur l'ensemble de la zone étudiée. En plus, ce logiciel nous permet de détecter avec précision l'apparition des fissures (figure 1).

Désignations des spécimens (B40-10 ou B80-14):

- La première partie de chaque notation (B40 ou B80) représente le $\mathrm{BO}$ ou $\mathrm{BHP}$,

- le chiffre (10 ou 14) correspond au diamètre d'armatures longitudinales.

Tableau 1. Proportions des bétons étudiés.

\begin{tabular}{|c|c|c|c|}
\hline Matériaux & Unités & BO & BHP \\
\hline E/C & - & 0,62 & 0,34 \\
\hline Ciment (CEMI-52.5 N) & $\mathrm{kg} / \mathrm{m}^{3}$ & 275 & 425 \\
\hline Fumée de silice & $\mathrm{kg} / \mathrm{m}^{3}$ & - & 42,5 \\
\hline Filler calcaire & $\mathrm{kg} / \mathrm{m}^{3}$ & 44 & - \\
\hline Eau & $\mathrm{L}$ & 170 & 144 \\
\hline Superplastifiant (Chrysofluid) & $\mathrm{L}$ & - & 6,4 \\
\hline Gravier (4/10) & $\mathrm{kg} / \mathrm{m}^{3}$ & 990 & 980 \\
\hline Sable (0/4 ) & $\mathrm{kg} / \mathrm{m}^{3}$ & 740 & 700 \\
\hline Affaissement & $\mathrm{cm}$ & 15 & 12 \\
\hline
\end{tabular}

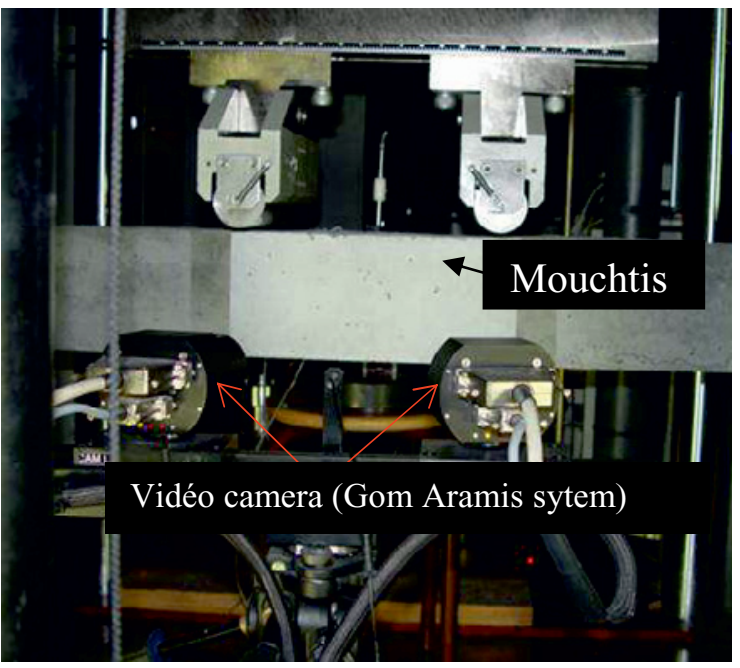

Fig. 1. Dispositif expérimental de l'essai de flexion.

\section{Résultats et discussions}

\subsection{Loi de comportement du matériau}

L'analyse du comportement non-linéaire des éléments de structure exige alors une connaissance très approfondie de comportement local du béton.

Sur la base des résultats expérimentaux (essais de compression), on se propose de modéliser la loi du comportement du matériau BHP. Celle-ci présente une branche pré-pic quasi-linéaire et une branche descendante post-pic très abrupte liée au caractère «fragile» de ce matériau (figure 2).

La courbe relative au béton ordinaire (BO) présente un assouplissement du matériau au delà de la moitié du chargement maximal (figure 2).

En comparant les courbes contrainte-déformation $(\sigma$ $\varepsilon)$ relatives aux $\mathrm{BO}$ et aux BHP, on s'aperçoit que l'augmentation de la compacité de la pâte de ciment dans le cas du BHP a eu pour effet direct d'étendre le domaine élastique des bétons BHP jusqu'à la résistance maximale (pic de contrainte). $\mathrm{Au}$ pic des contraintes, le raccourcissement du matériau se situe entre 0.0025 et 0.003 .

La déformation au pic de contrainte $\left(\varepsilon_{\mathrm{c} 0}\right)$ peut être évaluée au moyen de la formule de lissage que nous proposons ci-dessous à partir des résultats expérimentaux:

$\varepsilon_{c 0}=0,00143\left(f_{c}^{\prime}\right)^{0,167}$

Où $60 \leq f_{c}^{\prime} \leq 100 \mathrm{MPa}$, le coefficient de corrélation est de $r^{2}=0,96$.

L'observation attentive des diagrammes $\langle\sigma-\varepsilon\rangle$ d'essais obtenus montre que la courbe est réductible à une droite jusqu'à approximativement $85 \%$ de la contrainte maximale. En outre, la déformation ultime $\left(\varepsilon_{\mathrm{cu}}\right)$ semble être atteinte à $3 \%$, puisque la branche descendante (post-pic) est très abrupte au-delà d'une déformation de cet ordre de grandeur (figure 2).

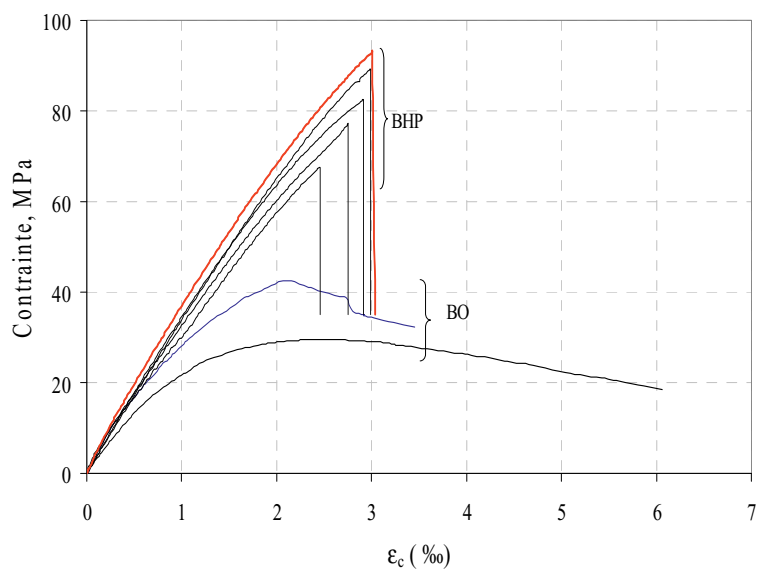

Fig. 2. Courbes $(\sigma-\varepsilon)$ d'essais obtenues.

Il semble donc plus approprié d'idéaliser la relation contrainte-déformation par un triangle-rectangle (diagramme bilinéaire) pour le BHP, avec un plateau de 
plasticité relativement réduit s'étalant de $2 \%$ à $3 \% 0$ comme en figure 3(a). Pour rappel, le diagramme idéalisé pour le béton ordinaire est donné en figure 3(b).

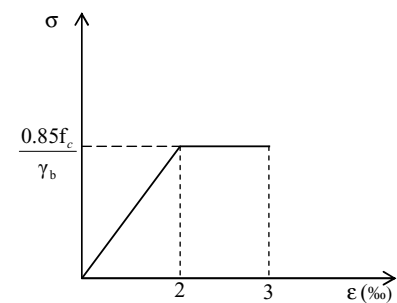

(a) BHP

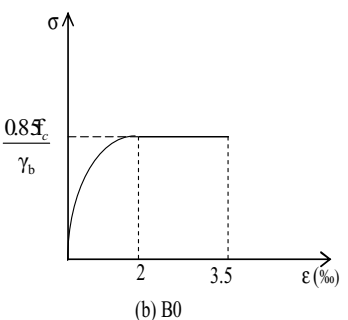

(b) B0
Fig. 3. Diagramme contrainte-déformation « $\varangle-\varepsilon »$.

\subsection{Flèches des poutres testées}

La capacité portante des poutres en B80 est élevée (6 à $20 \%$ ) que celle des poutres en B40 (figure 4). Ceci a pu être en effet considéré comme faible augmentation par rapport au doublement de la résistance à la compression de béton (de 40MPa à $85 \mathrm{MPa}$ ). Deux raisons expliquent cette faible augmentation. Tout d'abord, le béton à hautes performances augmente la résistance à la traction, mais réduit la profondeur de la zone comprimée. La seconde raison est liée au mécanisme de l'effet d'engrènement qui est absent dans les bétons à hautes performances, puisque la fissure traverse complètement les granulats, produisant une surface relativement lisse. C'est pour ces deux raisons que la capacité en flexion d'une poutre en B80 n'augmente pas de manière significative [3].

A la charge ultime, les flèches moyennes des poutres en B80 sont approximativement $22 \%$ plus importantes que celles des poutres en B40 (figure 4).

Par ailleurs, la difficulté concernant le calcul des flèches des poutres en béton armé est due aux incertitudes d'évaluation de la rigidité EI. L'expression (2) de la flèche illustre clairement que le problème particulier pour les structures en béton armé est la détermination de la rigidité en flexion EI d'un élément.

$\Delta_{c a l}=\frac{M_{a}}{24 E_{c} I_{e}}\left(3 L^{2}-4 a^{2}\right)$

où $\mathrm{M}_{\mathrm{a}}$ : le moment max. appliqué au milieu de l'élément, $\mathrm{E}_{\mathrm{c}}=4700 \sqrt{\mathrm{f}_{\mathrm{c}}^{\prime}}(\mathrm{MPa})$ : module d'élasticité à court terme

Le règlement Américain [4] recommande un moment d'inertie effectif comme étant une inertie comprise entre la valeur correspondant à la section fissurée équivalente $\left(\mathrm{I}_{\mathrm{cr}}\right)$ et celle correspondant à la section non-fissurée équivalente $\left(\mathrm{I}_{\mathrm{g}}\right)$, et est défini comme suit:

$$
\begin{aligned}
& I_{e}=\left(\frac{M_{c r}}{M_{a}}\right)^{3} I_{g}+\left[1-\left(\frac{M_{c r}}{M_{a}}\right)^{3}\right] I_{c r} \\
& M_{c r}=\frac{f_{r}}{y_{t}} I_{g}
\end{aligned}
$$

Où : $f_{r}=0,62 \sqrt{f_{c}^{\prime}}(\mathrm{MPa}), \mathrm{y}_{\mathrm{t}}=\mathrm{h} / 2$

Faza et Ganga Rao [5] ont établi une expression suivante donnant le moment d'inertie effectif.

$I_{m}=\frac{23 I_{c r} I_{e}}{8 I_{c r}+15 I_{e}}$

Ie et Icr sont donnés par l'équation (3).

Ashour [6] propose l'équation suivante, en tenant compte du taux d'armature longitudinale $(\rho)$ et la résistance à la compression de béton $\left(f_{c}^{\prime}\right)$ :

$$
\begin{aligned}
& I_{e}=\left(\frac{M_{c r}}{M_{a}}\right)^{m} I_{g}+\left[1-\left(\frac{M_{c r}}{M_{a}}\right)^{m}\right] I_{c r} \\
& m=3-0,8 \rho \sqrt{\frac{33}{f_{c}^{\prime}}}
\end{aligned}
$$

Les trois méthodes pour calculer le moment d'inertie effectif, exposées ci-dessus, ont été également utilisées avec Eq. (2) pour calculer la flèche des poutres testées.

Le tableau 2 donne les valeurs de la flèche prédites par les 3 méthodes à la charge de service, prise égale à 0,59 fois la charge ultime expérimentale [7].

A l'issue du tableau 2, il est clair que la variation de la flèche entre les valeurs expérimentales et les valeurs prédites par l'équation (5) est très faible. Cependant,

\begin{tabular}{|c|c|c|c|c|c|c|c|}
\hline \multirow{2}{*}{ Poutre } & \multirow{2}{*}{ Fexp } & \multicolumn{3}{|c|}{ Flèches calculées } & \multicolumn{3}{|c|}{ Fexp./Eq } \\
\hline & & Eq.3 & Eq.5 & Eq.6 & Eq.3 & Eq.5 & Eq.6 \\
\hline B40-10 & 4,15 & 3,65 & 3,75 & 3,64 & 1,14 & 1,11 & 1,14 \\
\hline B40-12 & 4,62 & 3,28 & 3,32 & 3,27 & 1,40 & 1,38 & 1,40 \\
\hline B40-14 & 4,74 & 4,18 & 4,19 & 4,18 & 1,13 & 1,13 & 1,13 \\
\hline B80-10 & 4,00 & 3,17 & 3,46 & 3,16 & 1,26 & 1,16 & 1,26 \\
\hline B80-12 & 4,13 & 3,29 & 3,40 & 3,29 & 1,22 & 1,18 & 1,22 \\
\hline B80-14 & 3,98 & 3,71 & 3,75 & 3,71 & 1,07 & 1,06 & 1,07 \\
\hline \multicolumn{5}{|c|}{ Moyenne (\%) } & 1,203 & 1,170 & 1,205 \\
\hline \multicolumn{5}{|c|}{ Ecart-type $(\%)$} & 0,107 & 0,102 & 0,108 \\
\hline \multicolumn{5}{|c|}{ Coefficient de variation $(\%)$} & 0,089 & 0,085 & 0,089 \\
\hline
\end{tabular}
l'équation 3 et l'équation 6 fournissent des résultats semblables.

Tableau 2. Flèches à la charge de service.

La Figure 4 présentant l'évolution des flèches mesurées et calculées en utilisant Eq. (2) et Eq. 5, en fonction des charges pour les deux types de béton (B40 et B80). 

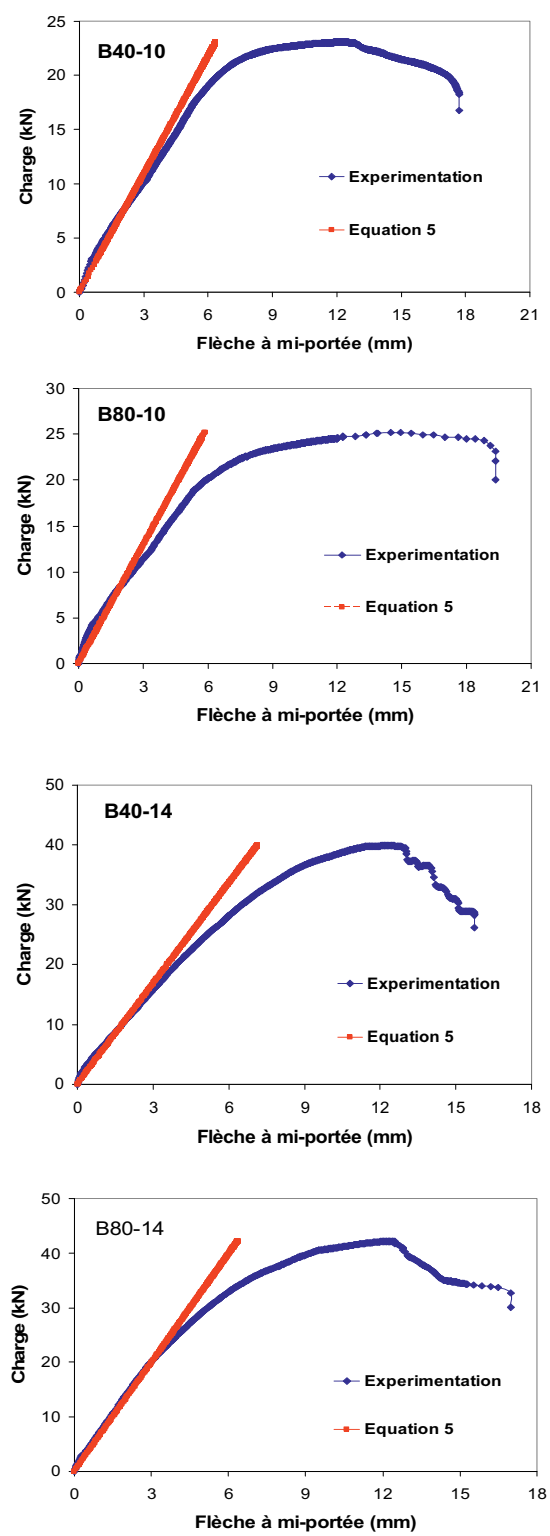

Fig. 4. Courbes charge-flèche de poutres testées.

L'analyse de la figure 4 montre clairement que l'équation (5) estime correctement la flèche jusqu'à la charge de service et ce pour les deux type de béton. Cependant, à des niveaux de chargement plus élevés (proche ou au delà de la charge ultime), une très grande différence entre les valeurs expérimentales et les valeurs prédites est constatée. Ceci est dû à la non-linéarité qui a lieu à des niveaux de charges élevées.

\subsection{Ductilité des poutres testées}

Si la rigidité, la charge et la flèche conditionnent le comportement structurel avant le pic de chargement, c'est uniquement la ductilité qui a une influence importante sur le comportement post-pic. La ductilité peut être définie comme étant la capacité d'une structure à se déformer plastiquement avant de se rompre. Cependant, il n'ya pas de consensus sur sa détermination; la plupart des règlements s'accordent à prendre comme définition le rapport de la flèche ultime $\left(\delta_{u}\right)$ sur la flèche correspondant à la limite élastique des armatures longitudinales $\left(\delta_{\mathrm{y}}\right)$. L'indice de ductilité est défini comme suit :

$\mu_{\delta}=\frac{\delta_{u}}{\delta_{y}}$

Les valeurs de l'indice de ductilité $\left(\mu_{\delta}\right)$ sont représentées sur la figure 5 .

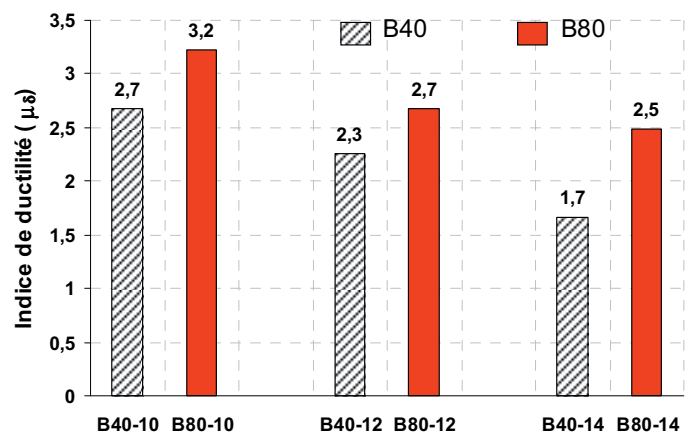

Fig.5. Indices de ductilité des poutres testées.

A partir de la figure 5, on remarque que pour le même pourcentage d'armatures longitudinales, la ductilité augmente lorsque la résistance à compression de béton augmente de 40 à $85 \mathrm{MPa}$. La valeur moyenne de $\mu_{\delta}$ pour les poutres en B80 est 1,30 fois celles des poutres en B40. Ceci indique que la ductilité d'un élément en béton armé est dépendante de la résistance à la compression de béton. Ceci n'est pas le cas quand le béton n'est pas armé où on remarque complètement l'inverse.

Cependant, la ductilité diminue lorsque le taux d'armature longitudinale augmente. La ductilité la plus élevée est observée pour les poutres à $\rho=1,22 \%$ et diminue en passant à $\rho=1,77 \%$ et à $\rho=2,42 \%$ ( $\rho$ croissant), pour les deux types de béton. Donc, pour un béton de nature donnée, il existe probablement un pourcentage d'armature pivot qui correspond à la ductilité maximale.

\subsection{Faciès de fissures et ouvertures de fissures}

Dans le cas des poutres en B80, on peut observer plusieurs fissures, bien réparties et uniformément ouvertes. (Figure 6). En conséquence, le nombre de fissures augmente lorsque la résistance du béton croît puisqu'on passe de 10 fissures pour B40-12 et de 14 fissures pour B80-12 Ceci est dû à une redistribution accrue des forces internes dans les poutres en B80.

En ce qui concerne les ouvertures de fissures, il est à noter que les ouvertures de fissures moyennes des poutres en B80 représentaient approximativement $80 \%$ celles des poutres en B40 [2].

Une des considérations importantes de conception à propos des éléments en flexion, est l'ouverture de fissures sous les charges de service. Dans ce sens, une étude comparative a été faite entre les valeurs expérimentales et les valeurs prédites par les trois modèles mathématiques 
de calcul des ouvertures de fissures, à savoir les modèles Américain (ACI318)[4], Britannique (BS8110)[8] et Européen (Eurocode 2)[9] (Tableau 3).
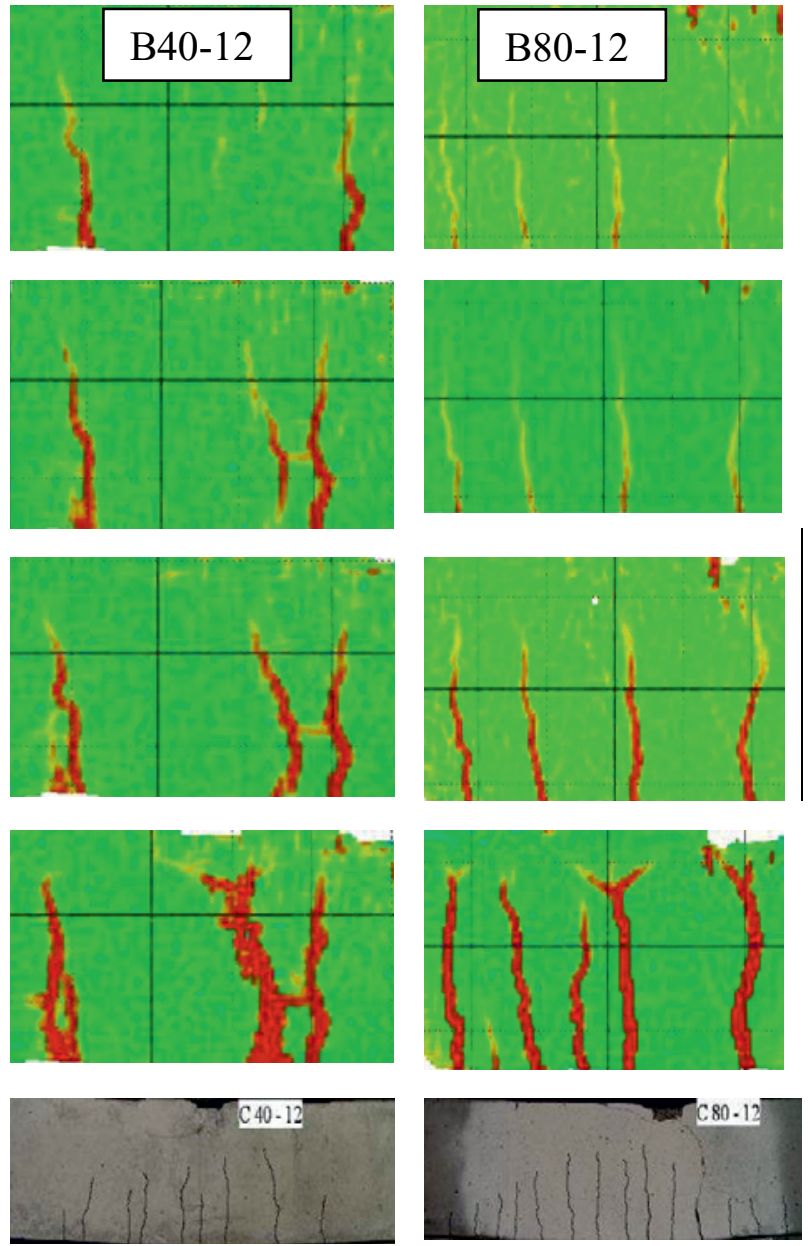

Fig. 6. Schématisation des étapes de fissuration des poutres (B40-12 et B80-12)

Le Tableau 3 illustre également que l'ouverture de fissure est influencée par la résistance à la compression de béton et le taux d'armature longitudinale. En fait, l'augmentation de la résistance du béton améliore l'adhérence acier-béton et donc réduit l'ouverture des fissures.

Tableau 3. Ouvertures de fissures (W) mesurées et calculées sous la charge de service.

\begin{tabular}{|c|c|c|c|c|c|c|c|}
\hline \multirow{2}{*}{ Poutre } & \multirow{2}{*}{$\mathrm{W}_{\text {test }}$} & \multicolumn{3}{|c|}{$\mathrm{W}_{\text {calculée }}$} & \multicolumn{3}{c|}{$\mathrm{W}_{\text {Tes }} \mathrm{t} / \mathrm{W}_{\text {calculée }}$} \\
\cline { 3 - 8 } & & $\mathrm{W}_{\mathrm{BS}}$ & $\mathrm{W}_{\mathrm{EC} 2}$ & $\mathrm{~W}_{\mathrm{ACI}}$ & $\mathrm{BS} 8110$ & $\mathrm{EC} 2$ & $\mathrm{ACI}$ \\
\hline B40-10 & 0,28 & 0,320 & 0,254 & 0,179 & 0,875 & 1,102 & 1,564 \\
\hline B40-12 & 0,25 & 0,271 & 0,203 & 0,150 & 0,923 & 1,232 & 1,667 \\
\hline B40-14 & 0,23 & 0,322 & 0,240 & 0,174 & 0,714 & 0,958 & 1,322 \\
\hline B80-10 & 0,22 & 0,345 & 0,261 & 0,190 & 0,638 & 0,843 & 1,140 \\
\hline B80-12 & 0,19 & 0,321 & 0,235 & 0,177 & 0,592 & 0,809 & 1,073 \\
\hline B80-14 & 0,18 & 0,339 & 0,245 & 0,183 & 0,531 & 0,735 & 0,984 \\
\hline \multicolumn{3}{|c|}{ Moyenne (\%) } & 0,712 & 0,942 & 1,292 \\
\hline \multicolumn{3}{|c|}{ Ecart-type (\%) } & 0,16 & 0,19 & 0,27 \\
\hline \multicolumn{3}{|c|}{ Coefficient de variation (\%) } & 22 & 20 & 21 \\
\hline
\end{tabular}

A l'issue du Tableau 3, on s'aperçoit que le règlement BS8110 surestime largement l'ouverture des fissures des poutres, particulièrement pour les poutres en B80. Par ailleurs, on constate que les valeurs prédites du règlement ACI318 sont proches des valeurs expérimentales et ce pour les poutres en $\mathrm{B} 80$.

Il faut également noter que la formule permettant le calcul de l'ouverture des fissures issue du règlement de l'Eurocode 2 prédit le mieux l'ouverture des fissures des poutres (pour l'ensemble des poutres testées). On note des ouvertures s'étalant entre $0,2 \mathrm{~mm}$ et $0,26 \mathrm{~mm}$, intervalle représentant les ouvertures de fissures usuelles sous les charges de service.

\section{Conclusions}

Les conclusions tirées de cette étude sont les suivantes:

- La capacité portante des poutres en BHP est élevée que celles des poutres en BO. Cette capacité est encore améliorée avec l'augmentation du taux d'armature longitudinale. Ce surcroît est de 6 à $20 \%$.

- Bien que le matériau BHP soit plus fragile que le BO. En présence, d'armatures, la ductilité du BHP armé s'améliore et devient meilleure que celle du BO armé. La valeur moyenne de l'indice de ductilité pour les poutres en BHP est 1,30 fois celles des poutres en BO.

- L'équation proposée par Faza and Ganga Rao semble mieux estimer la flèche jusqu'à la charge de service et ce pour les deux type de béton. Cependant, à des niveaux de chargement plus élevés (proche ou au delà de la charge ultime), une très grande différence entre les valeurs expérimentales et les valeurs prédites est constatée.

- A l'état limite de service, les ouvertures de fissures sont plus faibles dans le cas du BHP. Le matériau est servicible même à des niveaux de chargement élevé, dépassant les $75 \%$ de la charge ultime. Les ouvertures de fissures moyennes des poutres en BHP sont approximativement $80 \%$ des poutres en $\mathrm{BO}$.

- Parmi les trois modèles mathématiques de calcul des ouvertures de fissures, celui recommandé par l'ACI318 et celui recommandé par l'Eurocode 2 semble être rationnels et prédisent correctement les ouvertures des fissures sous les charges de services. Le modèle de BS8110 est plus conservatif et donc conduit à des ouvertures de fissures exagérées sous les charges de service et donc à des conceptions non économiques.

\section{Références}

1. LR. Taerwe, Brittleness versus ductility of High Strength Concrete. Structural Engineering, vol.1, $\mathrm{n}^{\circ} .4,(1990)$, pp. 40-45.

2. M. Hamrat, Comportement structural du béton à hautes performances (flexion et effort tranchant), Thèse de doctorat, Université USTHB-Alger, (2010). 
3. M. Hamrat, B. Boulekbache, M. Chemrouk. S. Amziane, Shear Strength of RC Beams without Stirrups Made of Normal Strength and High Strength Concretes", Advances in Structural Engineering, vol. 13, n'1, (2010), pp. 29-42.

4. Committee 318-05., Building Code Requirements for Structural Concrete (ACI 318-05) and Commentary (318R-05), American Concrete Institute, Farmington Hills, MI, 2005.

5. SS Faza, Ganga Rao., Fiber composite wrap for rehabilitation of concrete structures. Proceedings of the Materials Engineering Conference 804, ASCE, (1994), p. 1135.

6. SA. Ashour, Effect of compressive strength and tensile reinforcement ratio on flexural behavior of high-strength concrete beams, Engineering Structures, vol. 22, $\mathrm{n}^{\circ}$. 5, (2000), pp. 413-423.

7. M.A. Rachid and M.A. Mansur., Reinforced High Strength Concrete Beams in Flexure, ACI Structural Journal, vol. 102, $\mathrm{n}^{\circ} .3$, (2005), pp. 462-471.

8. BS8110., Structural Use of Concrete, Part1, Code of Practice for Design and Construction, British Standards Institution, London, 1997.

9. Eurocode 2., Calcul des structures en béton -Partie 11: Règles générales et règles pour les bâtiments, ENV 1992-1-1, 2004 